Bull. Mater. Sci., Vol. 3, No. 3, November 1981, pp. 247-253, e Printed in India,

\title{
Skull melting as a technique in the growth of single crystals
}

\author{
H R HARRISON and $\mathbf{J} M$ HONIG \\ Central Materials Preparation Facility, Department of Chemistry, Purdue \\ University, West Lafayette, Indiana 47907, USA
}

MS roceived 21 April 1981

\begin{abstract}
The growth of single crystals of many oxides is very difficult because of their high melting points, and in some cases, because of their extreme corrosiveness. Skull melting makes possible the preparation of many oxides because it is a crucibleless process, there is no theoretical limit as to the temperature that can be attained, and there is no intrinsic limitation on the oxygen pressure that may be used. A detailed description of how to set up a skull melting system and a detailed operating procedure are provided.
\end{abstract}

Kcywords. Crystal growth; skull melting; crucibleless; rf heating.

\section{Introduction.}

The objective of this paper is to draw attention to a technique that has been relatively recently developed for growth of a variety of single crystals, principally oxides. The procedure is based on the skull melting operation: it has several advantages over other techniques: (i) The process is crucibleless; a thin skull separates the molten mass from the water-cooled container. Thus, contamination problems or exsolution processes associated with contact between melts and hot crucibles are avoided. (ii) Single crystals of considerable size and perfection may readily be grown in copious quantities. (iii) It is readily possible to control the ambient, by use of gaseous buffers or by other means that fix the partial pressures of reactive gases over a very wide range; thus, one can control the stoichiometry of the material to be grown. (iv) It is an extremely versatile method: many different types of crystals may be prepared by this procedure.

The one drawback to this scheme is that it requires a rather high initial outlay of funds for capital equipment items; on the other hand, once the device is operational very little money is needed for maintenance and the equipment remains operational for many years.

The technique was originated in the USSR and France and further developed in the USA. Appropriate references to the original work are furnished below.

The procedure involves the coupling of a $250 \mathrm{~g}$ to $2 \mathrm{~kg}$ charge to a radio frequency electromagnetic field that is generated by a power supply operating lypically at $20-50 \mathrm{~kW}$ and usually in a dual range of frequency, namely 
250-750 $\mathrm{kHz}$ and 2-4 $\mathrm{MHz}$. The material is placed in a container of the type depicted in figure 1 (Wenckus 1977). It consists of a set of watercooled cold fingers set in a water-cooled base; the space between the fingers is sufficiently wide to permit penetration of the electromagnetic field into the interior, yet sufficiently small to prevent leakage of the melt from the interior to the outside. This, as well as the split-base arrangement, also avoids generation of large eddy currents in the copper metal.

Power transfer occurs via a water-cooled coil of several loops that surrounds the container. Usually, the resistance of the charge is too high to initiate the coupling to the work coil. It is therefore common practice to insert a ring of metal of the same material as forms a constituent in the final melt. The metal couples to the applied field and becomes sufficiently hot to heat adjacent portions of the charge to the point where they begin to couple to the field, thus generating further hot zones that couple and then spread to more remote portions of the charge. Alternatively, when an oxidizing atmosphere can be tolerated (even rather low oxygen partial pressures of $c a 10^{-12}$ atm are sufficient for the purpose) a graphite disk may be used as the starting susceptor. The disk ultimately floats to the top of the melt and 'burns off' to form CO and $\mathrm{CO}_{2}$ gas; this avoids the problem encountered with the denser metals that tend to sink to the bottom of the melt and become imbedded in the charge. The process is restricted to materials that are poor conductors at room temperature, so that a skull of sintered material can form in the vicinity of the cold fingers, thereby serving as the actual container for the molten charge.

In many cases it is necessary to control the composition of the melt by use of buffered gases such as $\mathrm{CO} / \mathrm{CO}_{2}$ for control of oxygen pressures, $\mathrm{H}_{2} / \mathrm{NH}_{3}$ for control of nitrogen pressures, etc. One must then have a very large volume of gas in an appropriate chamber above the sample so that all of the melt can be properly equilibrated.

Once the entire mass of material is melted and rendered uniform by convectional stirring the material is gradually lowered out of the stationary work coil in a modified Bridgman process which ultimately produces three types of regions in the final frozen boule: An outer, particulate layer of denselysintered material, an inner layer of needle-like or irregularly-shaped set of particulate aggregations, and a middle portion of single crystals. The top and bottom quarters of the boules obtained in this process are generally of poor quality and are discarded. A typical boule of $\mathrm{Fe}_{3} \mathrm{O}_{4}$ obtained by this process is shown in figure 2. The entire process may be completed in a normal working day.

\section{Experimental details}

Additional experimental details are provided below to assist individuals interested in reproducing the experimental set-up and procedure.

By far the most expensive piece of equipment required is a commercial radio-frequency generator, of the type manufactured by Lepel High Frequency Laboratories. A dual frequency (nominally, $450 \mathrm{kHz}$ and $3 \mathrm{MHz}$ ) machine 


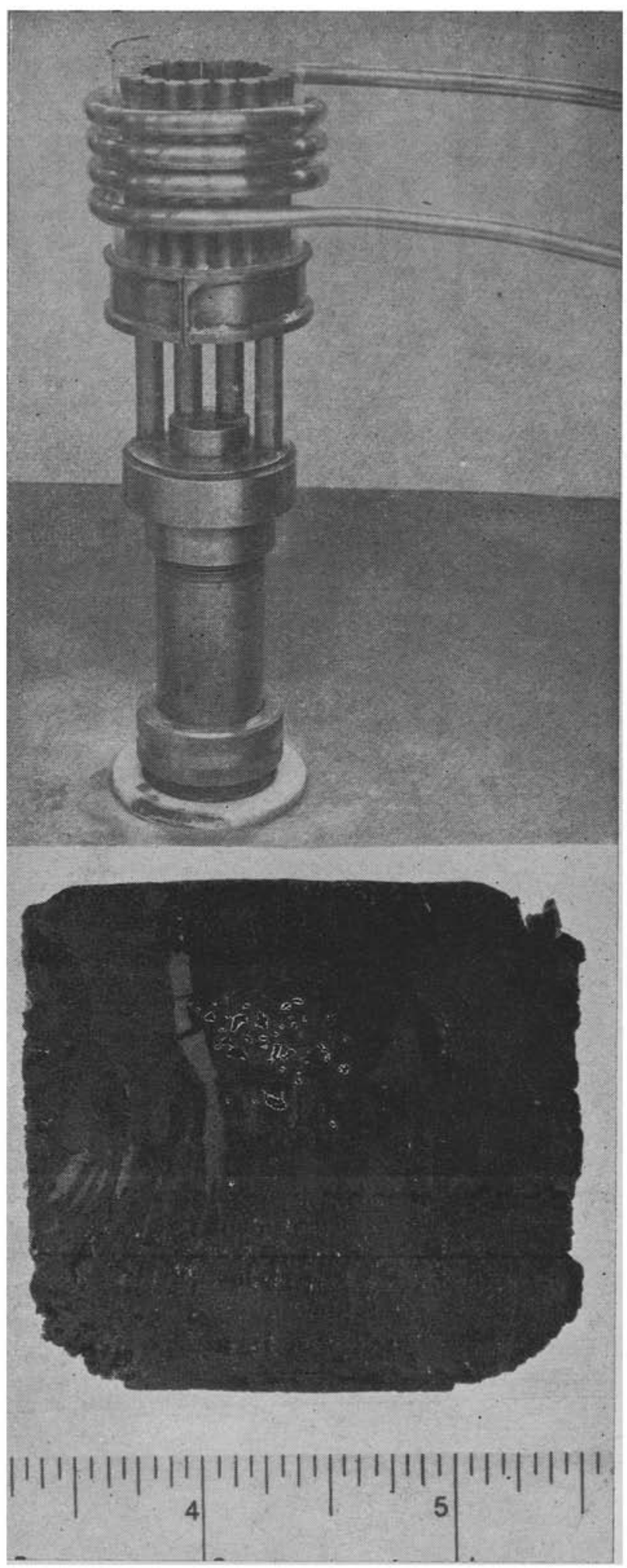

(1)

Figure 1. Cold crucible, quartz sleeve and work coil assembly used in skull melting experiments.

Figure 2. Interior of a boule produced by the skull melting technique, Note that individua! crystals may be clearly seen. Boule diameler (left to right) ahout $75 \mathrm{~mm}$. 


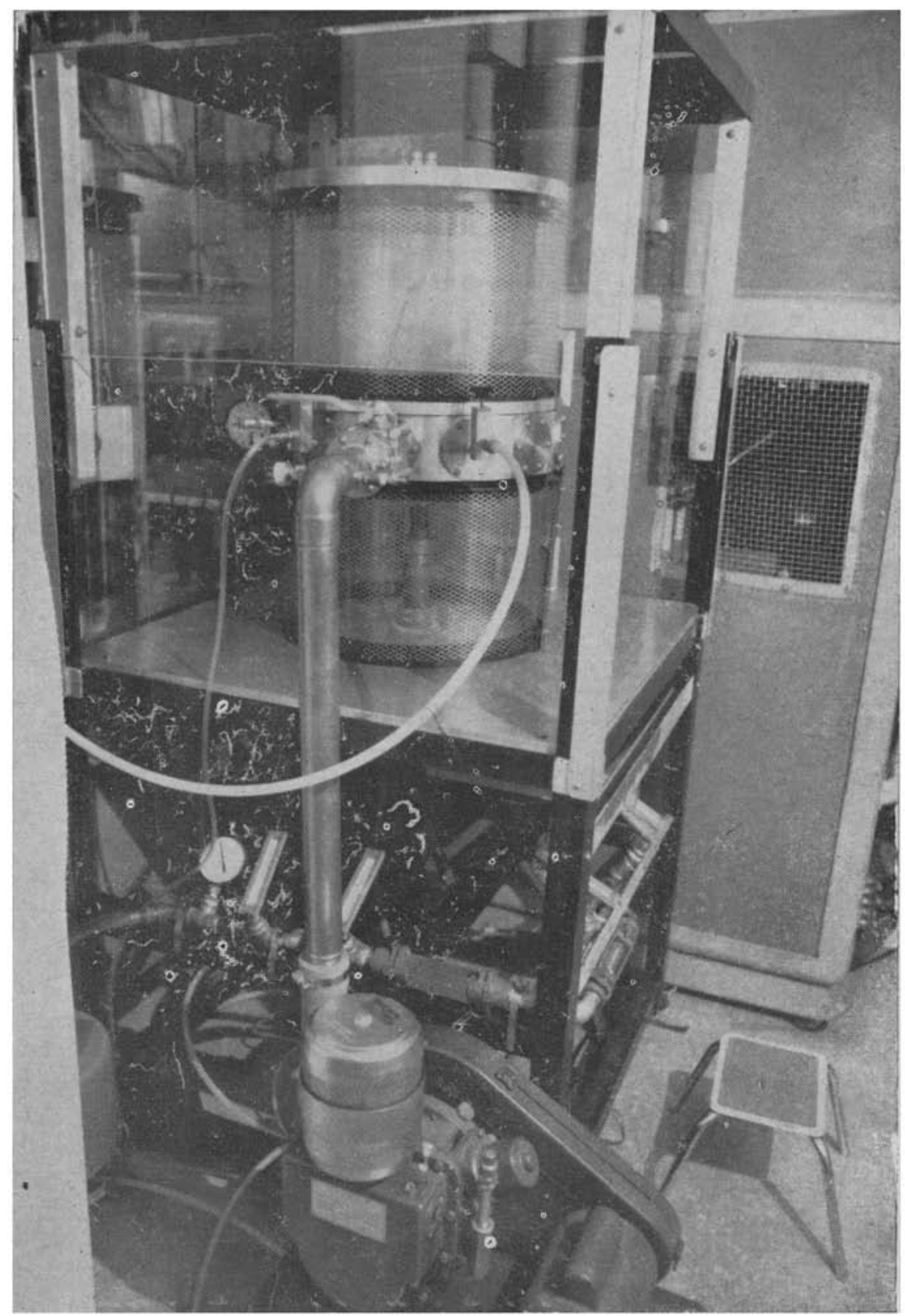

Figure 4. Lateral view of the skull melter airtight chamber. 


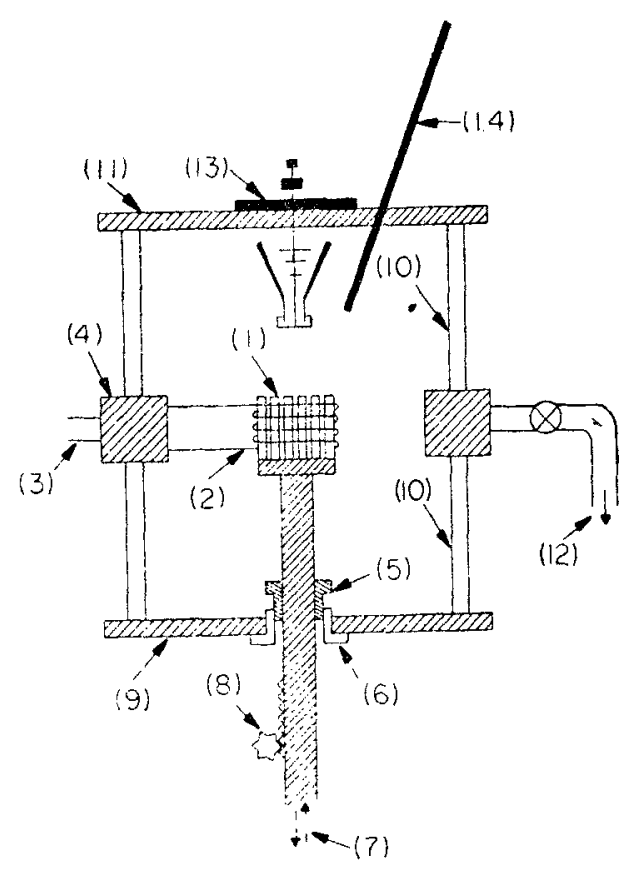

Figure 3. Schematic diagram of the skull melter as used in controlled atmosphere experiments. (1) Skull crucible; (2) work coil; (3) to rf generator; (4) 12-port vacuum collar; (5) vacuum quick-connect coupling; (6) Teflon insulating flanges; (7) water supply (in and out) for crucible; (8) motor driven gear system for lowering the crucible; (9) base plate and supporting frame; (10) 18" dia $\times 12$ " high pyrex vacuum cylinders; (11) aluminum top plate; (12) to mechanical pump; (13) powder hopper; (14) ceramic poker.

is preferred for research work; the rated power output should be at least $30 \mathrm{~kW}$; units used commercially range up to $100 \mathrm{~kW}$.

Figure 3 represents a schematic diagram of the skull melter system, and figure 4 shows a photograph of the actual apparatus. Everything shown in figure 3 is mounted inside a frame which also is attached to a hood for removal of noxious vapours.

To avoid excessive power loss, the frame is positioned so that the leads from the generator are as short as possible. These and the work coil are made of ordinary copper tubing; the latter must be carefully polished to remove even the smallest scratches, in order to avoid arc breakdowns. Of the items shown in figure 3 , only the vacuum quick connect coupling, the synchronous lowering motor, the pyrex vacuum cylinders, and the lowering motor were commercially purchased - all the other parts were made in shops at Purdue University, including the skull crucible itself. It is suggested that the top plate and multi-port vacuum collar be made of aluminum to minimize the weight of movable parts. An ordinary large-capacity mechanical pump is sufficient to 
produce a satisfactory vacuum preparatory to flowing in buffering gas mixtures such as $\mathrm{CO} / \mathrm{CO}_{2}$, which are premixed using a Matheson gas proportioner. Gauges for monitoring the influx and outflow water temperatures and the flow rates allow the experimenter to make sure the flow of cooling water to the skull melter is satisfactory, and also to calculate the power being carried away by the water.

Each of the "fingers" of the cold crucible shown in figure 1 has a smaller tube inside it for individual water cooling. There are individual chambers in each half of the split base for incoming and outgoing water. All parts are machined of copper, for good thermal conductivity. The four tubes coming out of the split base and the lower base, are made of brass for greater strength. All joints (except at the lower base) are silver-soldered. Also shown in figure 1 is the support column (which also carries the cooling water) onto which the skull crucible is screwed. Lower down is shown the vacuum quickconnect (via an o-ring seal) which allows the crucible to be lowered without loss of atmosphere control. Finally, the teflon insulating flange is shown, which allows the crucible to float electrically, thus avoiding arcing problems, which are a constant concern in the intense electromagnetic fields being employed. Also shown is the highly polished work coil (copper tubing) and a quartz insulating sleeve between it and the skull crucible. Four skull crucibles of different sizes have been constructed with capacities ranging roughly between $250 \mathrm{~g}$ and $2 \mathrm{~kg}$.

The procedure followed in the course of a typical run involves loading both the crucible and powder hopper. In addition, a graphite disk is placed in the crucible to act as a susceptor. The chamber $(\sim 125$ litre $)$ is then closed, evacuated $)$, and filled with appropriate buffering atmosphere, which is allowed to continue flowing through the system at the rate $10 \mathrm{litre} / \mathrm{min}$. Electromagnetic power is applied; when the initial charge is completely melted, more material is added from the powder hopper, which is operated without loss of atmosphere control, from the exterior via a vacuum-tight "quick-connect" fitting. The ceramic poker (which can similarly be operated without loss of atmosphere control) is used to break "bridges" of sintered material which may form during the early phases of melting. Once a melt of sufficient volume has been achieved, it is allowed to equilibrate with the atmosphere for an hour. The crucible is then slowly lowered (typically at a rate of $15 \mathrm{~mm} / \mathrm{hr}$ ) out of the stationary work coil by a synchronous motor-driven gear system until the whole charge is frozen. The chamber subsequently is opened, the skull crucible is unscrewed from its supporting water pipe, and the boule removed. The boule is then typically cut in half with a large diamond wheel saw, and the pieces are polished so that the various interior regions may be distinguished by visual examination. A sample of material from that portion of the boule that appears satisfactory may then be removed for further characterization by $x$-ray powder pattern analysis, polarized reflected light microscopy, and electron microprobe analysis.

\section{Acknowledgements}

One of the authors (HRH) acknowledges the support on NSF-MRL Grant DMR 77-23798 and the other (JMH) for the support in part on NSF Grant DMR 79-06886. 
The articles listed below in the references describe further details and the general methodology of the skull melter operation.

\section{References}

Aleksandrov V I, Osiko V V, Prokhorov A M and Tatarintsev V M 1973 Vestn. Akad. Nauk $S S R 1229$

Aleksandrov V I, Osiko V V, Prokhorov A M and Tatarintsev V M 1978 in Current opics in material science (ed.) E Kaldis (Amsterdam: North Holland Press) 1421

Harrison H R and Aragon R 1978 Mater. Res. Bull. 131097

Harrison H R, Aragon R and Sandberg C J 1980 Mater. Res. Bull. 15571

Michel D 1972 Rev. Int. Hautes Temp. Refract. 9225

Michel D, Perez Y J M and Collongues R 1978 J. Cryst. Growth 43546

Wenckus J F, Cohen M L, Emslie A G, Menashi W P and Strong P F 1975 Final Tech. Rep. AFCRL-TR-75-0213 